\title{
Hybrid and intermittent drying of carrot (Daucus carota var. Nantes)
}

\section{Szadzińska, J.; Mierzwa, D.*; Bukowski, K.}

Department of Process Engineering, Poznań University of Technology, Poznań, Poland *E-mail of the corresponding author: dominik.mierzwa@put.poznan.pl

\begin{abstract}
A combination of high power airborne ultrasound and microwaves in hot air drying was developed to investigate the effect of intermittent mode on process effectiveness and quality of carrot. The drying experiments were carried out in an innovative hybrid dryer. The course of moisture ratio, drying rate, specific energy and water consumption, colour, water activity and shrinkage were discussed. The results showed that intermittent ultrasound and microwaves in convective drying accelerate the heat and mass transfer, leading to shorter drying time and faster drying rate. It was found that hybrid-intermittent drying can improve the energy efficiency and product quality.
\end{abstract}

Keywords: intermittent drying; ultrasound; microwaves; energy; shrinkage. 


\section{Introduction}

Current trends in industrial equipment design or engineering in general place emphasis on the ecological aspect of production. Drying as a food processing method is particularly a good field for engineers and scientists to implement new or improve existing designs. Its most widely used method, hot air drying, is notorious for its bad energy efficiency (a result of constant drying agent replacement, necessary to finish the process) and long drying time. ${ }^{[1]}$ What is more, consumers' dietary knowledge is on the rise, which means that product quality should be taken into consideration as well. In that matter, convective drying also has its drawbacks, as it has a substantial impact on the dried foodstuffs' appearance, flavor and nutrients content. ${ }^{[2]}$ In latest years some ideas how to improve drying and driers have been reported. Among others, they include hybrid techniques and drying at nonstationary (intermittent) conditions. Former's principle of operation is a simultaneous use of two or more drying methods (heat sources), such as hot air and microwaves. In such a way, different mechanisms of energy transfer are employed, what makes the drying process faster and more energy-efficient. Latter, is based on providing a variable amount of heat during the process, so there are periods of intensive evaporation and so called relaxation, when moisture and temperature gradients inside the dried material are reduced. That results in reduction of thermal energy and better product quality, however it may also increase the drying time. ${ }^{[3,4]}$

In this research, carrot was selected to be an example of a foodstuff. Its distinctive orange color and shape, good nutritious value (content of dietary fiber, vitamins A, K, B6, carotenoids, lutein, zeaxantin) allowed it to take a place in various cuisines. ${ }^{[5,6]}$ Carrots are consumed in various forms, for example raw, boiled, soups, juices or even a cake. Furthermore, when dried, it can be used as an ingredient for dried vegetable mixes. In this paper, a novel drying technique involving hybrid methods in intermittent conditions is investigated. Both of those concepts examined alone proved to improve all three of crucial properties of drying: kinetics, energy consumption and product quality.

\section{Materials and Methods}

Carrot roots (Daucus carota, var. Nantes) used for the drying experiments were purchased from a local market and stored for at least $24 \mathrm{~h}$ at $4^{\circ} \mathrm{C}$. Before drying, the carrots were rinsed, peeled and cut into cubes (1.5 cm side). Next, $50 \mathrm{~g}$ carrot samples were dried in a hybrid dryer ${ }^{[7]}$, using eight different drying programs (Fig. 1). 


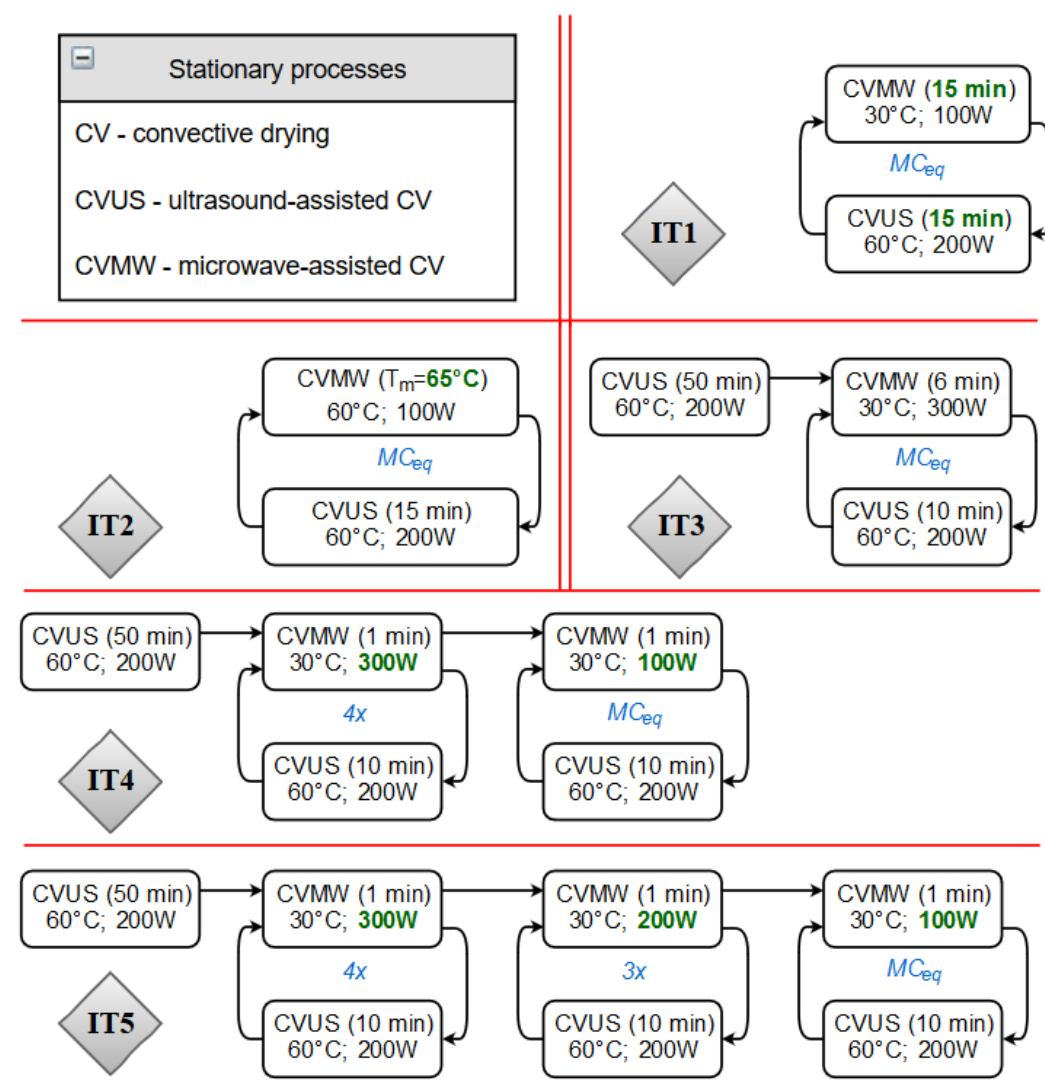

Fig. 1. Schematic illustration of the course drying programs used in the studies:

$T_{m}$ - material temperature, $M C_{e q}$ - equilibrium moisture content, $4 x$ - number of loop repetitions.

The drying processes were realized in stationary and non-stationary conditions. The stationary ones (CV, CVMW, CVUS) were conducted at the same air temperature $\left(T_{a}=60^{\circ} \mathrm{C}\right)$ with a constant air velocity $\left(\mathrm{v}_{\mathrm{a}}=2 \mathrm{~m} / \mathrm{s}\right)$, and with a changeless microwave or ultrasound power ( $\mathrm{P}_{\mathrm{mw}}=100 \mathrm{~W}-\mathrm{CVMW}$; $\left.\mathrm{P}_{\mathrm{us}}=200 \mathrm{~W}-\mathrm{CVUS}\right)$. The intermittent processes (IT1-5) were carried out in variable drying conditions (Fig. 1).

\subsection{Process kinetics and energy consumption}

The drying kinetics was assessed on the basis of the moisture ratio (MR), drying rate (DR), drying time (DT) and the specific energy consumption (SEC). The moisture ratio (MR) was determined from the following equation: 


$$
M R=\frac{M C_{t}-M C_{e q}}{M C_{i}-M C_{e q}}
$$

where: $\mathrm{MC}_{\mathrm{i}}, \mathrm{MC}_{\mathrm{t}}$ and $\mathrm{MC}_{\mathrm{eq}}$ are the initial, instantaneous (for a given time of the process) and equilibrium moisture content.

The initial moisture content $\left(\mathrm{MC}_{\mathrm{i}}\right)$ of the carrot was determined with the moisture analyzer (XM120; Precisa, Switzerland; precision 0.01\%). The equilibrium moisture content ( $\mathrm{MC}_{\mathrm{eq}}$ ) was constant during the research and equaled $0.05 \mathrm{~kg} \cdot \mathrm{kg}^{-1} \mathrm{db}$. The moisture content at a given time of the process $\left(\mathrm{MC}_{\mathrm{t}}\right)$ was expressed as the ratio of moisture mass to the initial mass of wet sample. The energy consumption was measured with a standard electricity meter (precision $0.01 \mathrm{kWh}$ ) and recalculated to MJ per $\mathrm{kg}$ of evaporated moisture (SEC). Because some devices in the drying system require tap water as a coolant, the specific water consumption (SWC), that is $\mathrm{dm}^{3}$ of water per $\mathrm{kg}$ of evaporated moisture, was also measured.

\subsection{Quality assessment}

The product quality was assessed on the basis of water activity, color, volumetric shrinkage and apparent density. The color of the fresh/dry carrot was measured with the use of colorimeter (CR400; Konica Minolta, Japan; precision 0.01). For this purpose, several randomly chosen (fresh/dried) samples were ground in a laboratory mill (A11; IKA; Germany). On the basis of the obtained CIE Lab tristimulus color coordinates ( $\mathrm{L}^{*}, \mathrm{a}^{*}$, and $\mathrm{b}^{*}$ ), the relative color change $(\mathrm{dE})$ was determined:

$$
d E=\left(d L^{* 2}+d a^{* 2}+d b^{* 2}\right)^{0.5}
$$

where: $\mathrm{dL}^{*}, \mathrm{da}^{*}$ and $\mathrm{db}^{*}$ are the differences between particular color coordinates $\left(\mathrm{L}^{*}, \mathrm{a}^{*}, \mathrm{~b}^{*}\right)$, obtained for the raw and dry material, respectively.

The water activity (aw) was measured for fresh/dried samples with the use of laboratory aw meter (LabMaster-aw; Novasina, Switzerland; precision 0.001) at constant temperature of the chamber $\left(25^{\circ} \mathrm{C}\right)$. The volumetric shrinkage of the carrot samples (VS) was determined with the use of modified toluene method developed by Mazza. ${ }^{[8]}$ The volumetric shrinkage was calculated with the following equation:

$$
V S=\left(1-\frac{V_{d}}{V_{0}}\right) \cdot 100 \%,
$$

where: $V_{d}$ and $V_{0}$ are the volumes of dry and fresh sample, respectively. 


\section{Results and discussion}

\subsection{Drying kinetics, energy and water consumption}

In Figure 1, the evolution of moisture ratio (MR) and the average drying rate (DR) for different drying processes were presented.
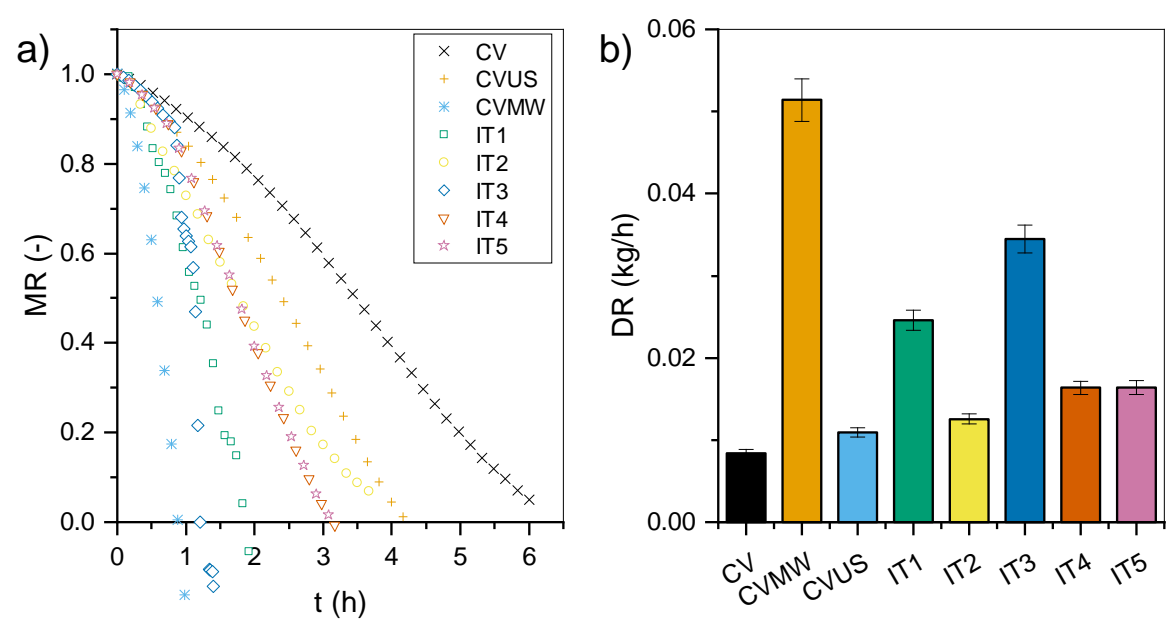

Fig. 1. Evolution of moisture ratio MR (a) and the average values of drying rate $D R$ (b).

As follows from figure 1, the convective drying (CV) was the slowest drying procedure and lasted about six hours. The application of microwave radiation during convective drying reduced the overall drying time by $85 \%$, as compared to CV. In turn, the ultrasound assistance in convective drying (CVUS) improved the drying kinetics, shortened the drying time by $32 \%$, and increased the drying rate by about $30 \%$ in comparison to CV. The total drying time for the samples dried by IT processes was in the range of 1-4 hours. Therefore, hybrid drying with intermittent MW and US application enabled to achieve the desired effect, i.e. increase in process efficiency.

Figure 2 shows the specific energy (SEC) and water consumption (SWC) for different drying strategies. The highest electric energy consumption was observed for CVUS, and the lowest for CVMW (Fig. 2a). Satisfactory results were obtained for intermittent drying. But the differences between the total energy consumed in the IT1-5 procedures were dependent on both durations of MW and US application. In turn, as follows from figure $2 \mathrm{~b}$, the longer MW radiation time the more water consumed in the drying process. The IT2-5 methods are characterized by much smaller and similar values of SWC than CVMW and IT1, which is a very positive effect. It means also, that modification of convective-microwave drying into intermittent mode ensures less water consumption. 

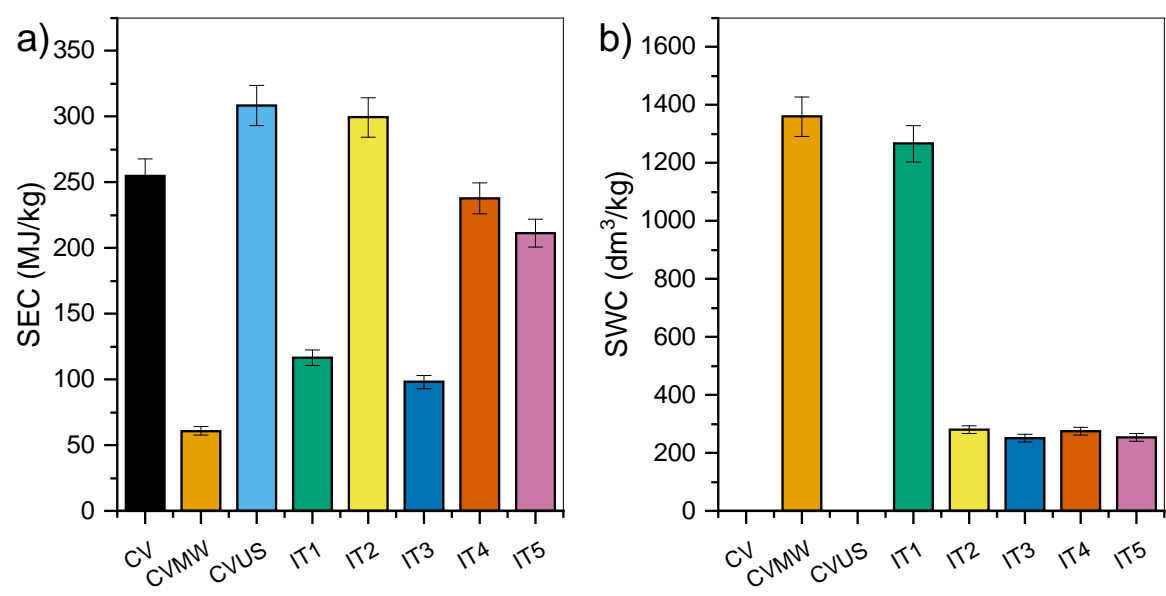

Fig. 2. Specific energy consumption SEC (a) and water consumption SWC (b).

\subsection{Quality characteristics}

After drying the carrot samples were assessed in terms of water activity (aw), color change $(\mathrm{dE})$, volumetric shrinkage (VS) and apparent density $\left(\rho_{\mathrm{a}}\right)$. Figure 3a presents the water activity of raw and dry material. As follows from this graph the aw value was reduced from about 0.989 (for fresh carrot root) to on average 0.333 for dry products.
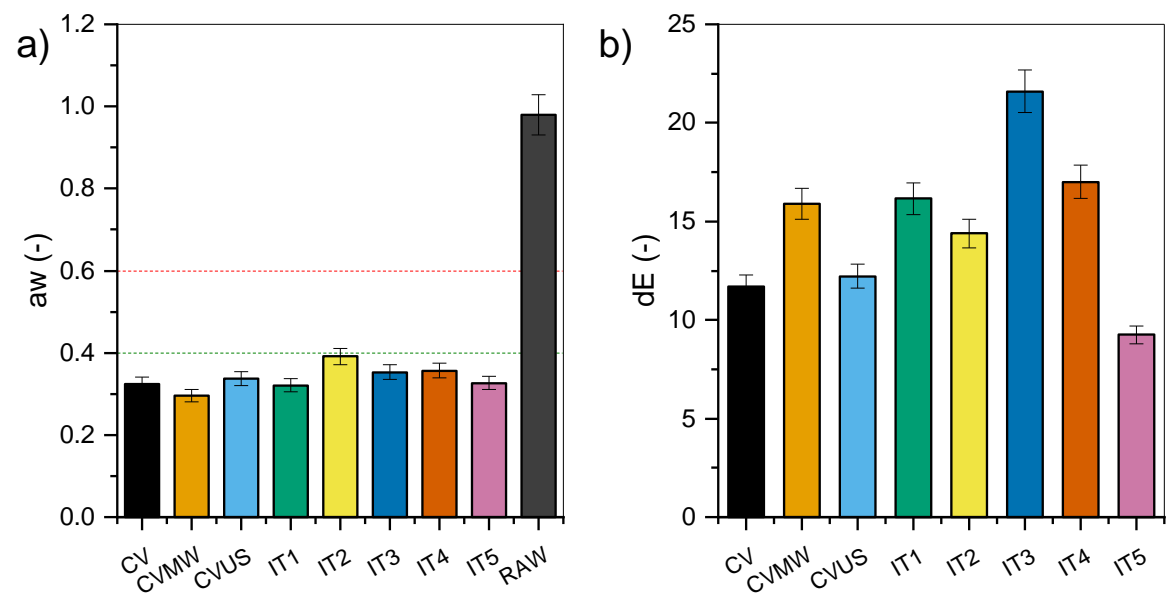

Fig. 3. Water activity aw (a) and relative color change $d E$ (b).

According to Labuza et al. ${ }^{[9]}$, when the water activity is below 0.4 the dried products are stable in terms of the growth of bacteria, yeast and molds, and natural decay processes (e.g. non-enzymatic browning). 
As follows from the data presented in figure $3 \mathrm{~b}$, the highest and the lowest relative color change was obtained for the samples dried by intermittent methods (IT3 and IT5 respectively). However, the other samples did not differ significantly, among specific drying programs, i.e. stationary and non-stationary. The exception is CVMW, for which the color change was close to those of IT.

The next quality factor assessed in the studies was volumetric shrinkage (Fig. 4a). On the basis of the results, it was found that the carrot samples dried by CV and CVUS shrank by $85 \%$, on average. In turn, the rest of the samples dried by CV combined with MW, were characterized by a lower shrinkage. This effect results from the so-called "puffing". The best shape retention was observed for the IT3 samples, but the value of VS is also fairly high. These results were confirmed by calculation of additional value, i.e. apparent density $\rho_{a}$ (Fig. 4b).
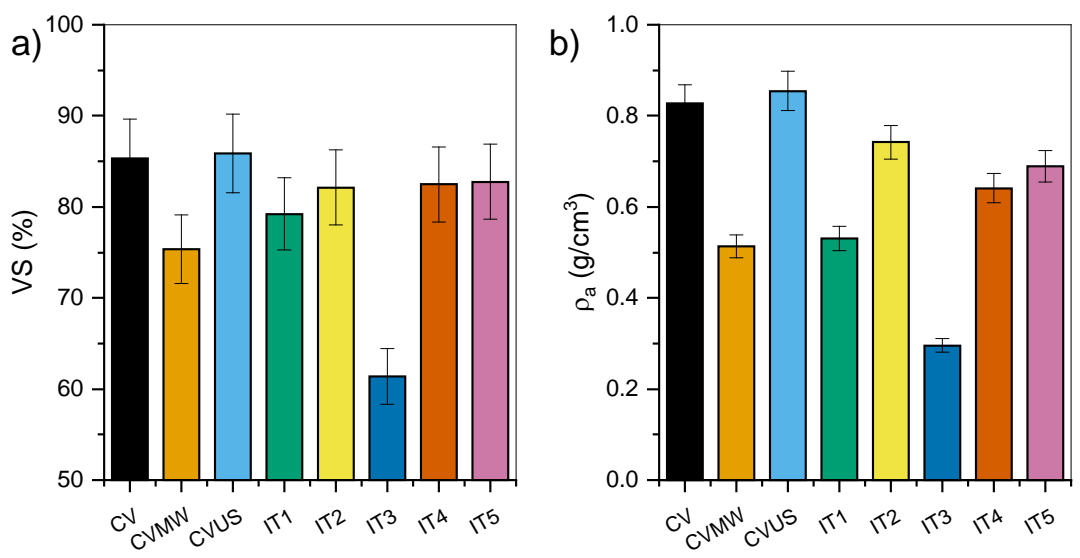

Fig. 4. Volumetric shrinkage VS (a) and apparent density $\rho_{a}(b)$.

The highest value of $\rho_{a}$ was observed for the CVUS drying whereas the lowest value of $\rho_{a}$ was observed for the IT3 drying. Lower values of $\rho_{a}$ means higher total volume of the sample, and thus a lower shrinkage after drying. Furthermore, a lower value of the apparent density means that dry sample is characterized by higher porosity (assuming same mass of samples). Therefore, the results of volumetric shrinkage and apparent density demonstrated, that the final physical and microstructural properties of the carrot samples depend on the drying technique and are better for simultaneous MW and US application in convective drying. 


\section{Conclusions}

The results showed that intermittent ultrasound and microwaves in hot air drying improve significantly the process efficiency as compared to the CV drying. Furthermore, it was demonstrated that intermittent drying can reduce both energy and water consumption as well as improves the quality of dry carrots. Adequate choice of process parameters positively affects the product color and its physical properties such as lower shrinkage (shape retention) and higher porosity.

\section{Acknowledgement}

This study was conducted as a part of research project no. 2014/15/D/ST8/02777 sponsored by the National Science Centre in Poland.

\section{References}

[1] Musielak, G. Advanced drying techniques; Poznań University of Technology: Poznań, 2013 (in Polish).

[2] Nowacka, M.; Śledź, M.; Wiktor, A.; Witrowa-Rajchert, D. Physical and chemical properties of microwave dried food products. ŻYWNOŚĆ. Nauka. Technologia. Jakość 2012, 6 (85), 5-20 (in Polish).

[3] Chua, K.J.; Mujumdar, A.S.; Chou, S.K. Intermittent drying of bioproducts—an overview. Bioresource Technology 2003, 90 (3), 285-295.

[4] Kowalski, S.J.; Szadzińska, J.; Łechtańska, J. Non-stationary drying of carrot: Effect on product quality. Journal of Food Engineering 2013, 118 (4), 393-399.

[5] U. S. D. o. Agriculture, „National Nutrient Database for Standard Reference,” [Online].

Available: https://ndb.nal.usda.gov/ndb/search/list?qlookup=11124\&format=Full.

[6] Alasalvar, C.; Grigor, J.M.; Zhang, D.; Quantick, P.C.; Shahidi, F. Comparison of volatiles, phenolics, sugars, antioxidant vitamins, and sensory quality of different colored carrot varieties. Journal of Agricultural and Food Chemistry 2001, 49 (3), 14101416.

[7] Kowalski, S.J.; Mierzwa, D. US-assisted convective drying of biological materials. Drying Technology 2015, 33 (13), 1601-1613.

[8] Mazza, G. Dehydration of carrots: Effect of pre-drying treatments on moisture transport and product quality. Journal of Food Technology 1983, 18, 112-123.

[9] Labuza, T.; Tannenbaum, S.; Karel, M. Water content and stability of low moisture and intermediate-moisture foods. Food Technology 1970, 24 (5), 543-550. 